\title{
Minimal Coupling of Electromagnetic Field in Riemann-Cartan Spacetime for Perfect Fluids
}

\author{
Larry L. Smalley ${ }^{1}$ and Jean P. Krisch ${ }^{2}$
}

Received August 23, 1991

\begin{abstract}
We minimally couple the electromagnetic field to gravity in Riemann-Cartan spacetime in the self-consistent formalism for perfect fluids by treating the internal energy of matter as a function of the electromagnetic field. The overall Lagrangian of the gravitational field, perfect fluid, and the electromagnetic field is constrained to be gauge invariant under gauge transformations of the vector potential. The theory preserves both charge conservation and particle number conservation, and gives the usual form of the free field equations.
\end{abstract}

\section{INTRODUCTION}

We consider the problem of "minimally" coupling the electromagnetic (EM) field to Riemann-Cartan (RC) spacetime for charged perfect fluids. One of the major historical problems with coupling the EM field to any spacetime geometry has been the maintenance of gauge invariance, of charge conservation in the theory (Novello, 1976). One solution to this problem has been the assumption that, in lifting the EM field from the arena of special relativity into a more complex geometry, the EM field tensor is taken to be a two-form (Benn, 1982; Heyl et al., 1976; Mukku and Sayed, 1979). It is well known that the generalization of a geometrical object to a more complex space can be done in many ways, i.e., there is no a priori requirement that the EM field be a two-form (Spinosa, 1987). One is free to impose prior constraints on the fields, such as in the two-form approach for the EM field, or overall constraints on the form of the field equations after generalization. The latter condition is equivalent to requiring that the Lagrangian satisfy certain conditions, in this case, overall gauge invariance. We will take this approach for coupling the EM field to RC spacetime.

\footnotetext{
'Department of Physics, University of Alabarna in Huntsville, Huntsville, Alabama 35899.

${ }^{2}$ Department of Physics, University of Michigan, Ann Arbor, Michigan, 48109.
} 
In our development, the self-consistent Lagrangian formalism for perfect fluids in RC spacetime (Ray and Smalley, 1982a, 1983a,b) forms the basic Lagrangian to which we add a Lagrangian for the EM field. We point out that earlier work by Amorim $(1984,1985)$ and by de Ritis et al. $(1985)$ using the self-consistent formalism assumes that the EM field is a two-form. Instead, here we assume that the EM field is minimally coupled to the geometry of RC spacetime in the sense that the flat-space derivatives are generalized to RC spacetime via the asymmetric connection of RC spacetime. The EM Lagrangian contains the usual field Lagrangian plus a current term (e.g., Amorim, 1984). It will be necessary to show how one is able to conserve gauge invariance, not only for the EM field tensor, but also for the Lagrangian as a whole.

Our treatment of a perfect fluid interacting with an EM field is closely related to that of the Einstein-Maxwell theory in Riemannian geometry in which the flat-space derivatives of Minkowski space are generalized via the Riemannian connection to the covariant derivatives of Riemannian geometry. It is purely an accident, due to the symmetry of the Christoffel connection, that this approach in general relativity coincides with the two-form approach for the electromagnetic field. In our self-consistent approach, all fields, including the electromagnetic field, are coupled to the geometry via the $\mathrm{RC}$ connection. The EM fields are thus considered part of the system and therefore mutually interact with all fields. By contrast, the two-form approach seems to treat the EM field as an external field.

In Section 2 we briefly review the self-consistent Lagrangian for a perfect fluid, and in Section 3 we introduce the EM Lagrangian. We discuss gauge invariance in Section 4. The complete Lagrangian and its variation are given in Section 5, and we derive the various field equations in Section 6. In Section 7, we demonstrate several of the technical details involving the field equations, such as charge conservation and the free field equations. We give our conclusions in Section 8.

\section{LAGRANGIAN FOR PERFECT FLUID}

The self-consistent Lagrangian formulation for perfect fluids has been described for both general relativity and RC spacetimes (Ray, 1972; Ray and Smalley, 1982b). This Lagrangian density is given by

$$
L=L_{G}+L_{F}
$$

where the Lagrangian densities for the gravitatjonal field and the perfect fluid are given respectively by

$$
L_{G}=e \mathscr{R} / 2 \kappa
$$


and

$$
\begin{aligned}
L_{F}= & e\left\{-\rho\left[1+\varepsilon\left(\rho, s, F_{i j}\right)\right]-\lambda_{2} \stackrel{*}{\nabla}_{i}\left(\rho u^{i}\right)+\lambda_{3} u^{k} \partial_{k} X+\lambda_{4} u^{k} \partial_{k} s\right. \\
& \left.+\lambda_{1}\left(g_{i j} u^{i} u^{j}+1\right)\right\}
\end{aligned}
$$

where $\mathscr{R}$ is the scalar curvature, $\kappa=8 \pi G, G$ is the gravitational constant, $g_{i j}$ is the metric tensor with holonomic indices given by Latin characters such that $i=0,1,2,3$, and $e=(-g)^{1 / 2}$, where $g=\operatorname{det}\left(g_{i j}\right)$. The matter density is $\rho$, $s$ is the entropy density, $X$ is the fluid parameter associated with the Lin constraint (e.g., Ray, 1972) and $u^{i}$ is the four-velocity of the fluid particles. The function $\varepsilon$ then represents the internal energy of the fluid, which is a function of the density, entropy and the EM field in the self-consistent formalism. The various $\lambda$ 's are the Lagrange multipliers that ensure particle number conservation, Lin constraint, conservation of entropy, and the orthonormality of the four-velocity. The "star" derivative is $\nabla_{i}=\nabla_{i}+2 S_{i}$, where $\nabla_{i}$ is the usual covariant derivative in RC spacetime with torsion tensor, $S_{i j}{ }^{k}=\Gamma_{[j]]}{ }^{k}$, and $S_{i}=S_{i x}{ }^{x}$. A discussion of the star derivative can be found in Hehl $(1973,1974)$. An overdot on a variable $a$ represents the action of derivatives along fluid flow lines, $u^{k} \nabla_{k} a \equiv \dot{a}$. The perfect-fluid Lagrangian is the generalization to RC spacetime of the general relativistic perfect-fluid Lagrangian density of Ray (1972).

The second leg of the self-consistent formulation is the thermodynamics for a perfect fluid,

$$
d \varepsilon=T d s-p d(1 / \rho)
$$

where $T$ is the temperature and $p$ is the pressure. After introducing the EM Lagrangian in the next section, we will generalize the thermodynamics to include EM contributions to the internal energy.

\section{LAGRANGIAN FOR CHARged, PERFECT FLUID}

We wish to extend the general form for the EM Lagrangian

$$
L_{E M}=e\left\{-\frac{1}{4} F^{i j} F_{i j}+J^{k} A_{k}\right\}
$$

where $F^{i j}$ is the EM field tensor, $J^{k}=q \rho u^{k}$ is the current four-vector, $q$ is the electronic charge, and $A^{k}$ is the four-vector potential of the EM field. However, we do not assume that the EM field tensor is a two-form. Instead we minimally couple the EM field to the geometry in the sense that

$$
F_{i j}=2 \nabla_{[i} A_{j]}
$$

If the EM field is coupled to the geometry of spacetime, then it, as well, should be treated as a thermodynamic variable in the self-consistent 
formulation. The thermodynamics of EM systems has been discussed extensively by Guggenheim (1936). Accordingly, for charged perfect fluids, we extend the thermodynamics from equation (4) to

$$
d \varepsilon=T d s-p d(1 / \rho)-(1 / 2 \rho) F^{i j} d F_{i j}
$$

By including the last term in equation (7), we include the energy density of all EM fields as part of the total system.

In the next section, we show how we must augment the EM Lagrangian in order to maintain gauge invariance.

\section{GAUGE INVARIANCE}

The idea behind gauge invariance in any Lagrangian-based theory follows the general approach for the Dirac Lagrangian. For Dirac fields, gauge invariance requires extending the usual gauge transformation of the vector potential to include a "gauge transformation of the second kind" (Messiah, 1962) for the quantum Dirac field. The equivalent field in RC spacetime is the torsion field. Thus we require that $F^{i j}$ (as well as $L$ ) be invariant under the extended gauge transformation (Smalley, 1986)

$$
\begin{aligned}
A_{j}^{\prime} & =A_{j}-\partial_{j} \varphi \\
S_{i j}^{\prime k} & =S_{i j}{ }^{k}+\frac{2}{3} b \delta_{[i}^{k} \partial_{j]} \varphi
\end{aligned}
$$

where $\varphi(x)$ is the gauge field and $b$ is a constant of proportionality. The equivalent effect on the connection is

$$
\Gamma_{i j}^{\prime k}=\Gamma_{i j}{ }^{k}+\frac{2}{3} b \delta_{i}^{k} \partial_{j} \varphi-\frac{2}{3} b g_{i j} \partial^{k} \varphi
$$

Previous attempts to generalize the Dirac field to RC spacetime only seems to have been successful if the torsion field is restricted to the torsion vector part (Mukku and Sayed, 1979; Hojman et al., 1978) without proper torsion. We do not make any a priori constraint on the proper torsion. In our case, any such restriction must follow naturally from the variation of the Lagrangian. We do note that the torsion vector seems to play an important role in these theories, and in some cases is assumed to be proportional to the vector potential itself (Hammond, 1988, 1989). In order to understand this aspect of our Lagrangian, we write the torsion tensor in terms of its proper (trace-free) and torsion vector (trace) parts

$$
S_{i j}^{k}=\hat{S}_{i j}^{k}-\frac{2}{3} \delta_{[i}^{k} S_{j]}
$$

where the caret indicates the proper torsion part of the torsion tensor. Combining equations (9) and (11), we see immediately that the extended gauge 
transformation of the torsion tensor is equivalent to the transformation

$$
\begin{aligned}
S_{i}^{\prime} & =S_{j}-b \partial_{j} \varphi \\
\hat{S}_{i j}^{\prime k} & =S_{i j}{ }^{k}
\end{aligned}
$$

By comparison with equation (8), one may conclude that the torsion vector and the vector potential could be proportional, i.e.,

$$
S_{j} \equiv b A_{j}
$$

We will therefore assume this restriction in the EM Lagrangian.

The combined action of the extended gauge transformation on the EM field gives

$$
F_{i j}^{\prime}=F_{i j}-2\left\{\frac{2}{3} b A_{[i} \partial_{j]} \varphi+\frac{2}{3} S_{[j} \partial_{i]} \varphi-\hat{S}_{i j}{ }^{k} \partial_{k} \varphi\right\}
$$

Normally the process of gauging the Lagrangian field and the variation of the Lagrangian are independent operations. However, here we have included the constraint (14) in our overall Lagrangian. This allows us to use the relationship between the torsion vector and the vector potential in the gauging process. The transformed EM field is now invariant if we further require that the proper torsion and the gauge satisfy the condition

$$
\hat{S}_{i j}{ }^{k} \partial_{k} \varphi=0
$$

It is not obvious at this point that we can justify this condition, since it seems at first that just the EM field tensor $F_{i j}$ given by equation (6) is a function of the proper torsion; in Section 6 we will discover that equation (16) is correct.

It is interesting to note that in some theories (Smalley and Ray, 1986) involving spin fluids, the proper torsion satisfies the field equation

$$
\hat{S}_{i j}{ }^{k}=\frac{\kappa}{2} \rho s_{i j} u^{k}
$$

In this case, the condition on the gauge would reduce to the requirement that the gauge in the comoving frame is not an explicit function of time, i.e.,

$$
u^{0} \partial_{0} \varphi=0
$$

Such a condition on the gauge, equation (18), is very similar to the harmonic condition imposed in classical electromagnetism when, for example, one imposes a particular gauge such as the Lorentz gauge. The same effect occurs for general relativity (Grensing and Grensing, 1983a,b). Thus we are led naturally to the addition of a gauge-fixing term to our EM Lagrangian (Grensing and Grensing, 1983a,b; Fock, 1964; Dirac, 1959).

Because of the above considerations, we could add a term to the EM part of the Lagrangian which fixes the Lorentz gauge in RC spacetime, but 
first we must determine whether we can also make the total Lagrangian invariant under gauge transformations as well. It is not difficult to determine that the perfect-fluid part of the Lagrangian is invariant, but the gravitational field part is not. First we write the scalar curvature in terms of its Riemannian plus torsion parts

$$
\mathscr{R}=K+4 \nabla_{k}^{\{} S^{k}-\frac{8}{3} S_{k} S^{k}+\hat{S}_{j k l} \hat{S}^{j k l}+2 \hat{S}_{j k l} \hat{S}^{j l k}
$$

where the scalar curvature $K$ and the covariant derivative $\nabla_{k}^{\{\}}$are those of Riemannian spacetime. Considering only the torsion vector terms, we note that the variation of the divergence term vanishes, but the variation of the torsion vector squared term does not vanish,

$$
\delta\left\{-\frac{8}{3} S_{k} S^{k}\right\}=-\frac{16}{3} S_{k} \delta S^{k}
$$

In order to maintain gauge invariance of the total Lagrangian, the correct form of the gauge-fixing term should be

$$
L_{\mathrm{GF}}=-e\left\{\frac{2 b}{3 \kappa} \nabla_{k} A^{k}\right\}
$$

Our form of the EM Lagrangian is found by combining equations (5) and (21) plus a (torsion vector)-(vector potential) constraint term following equation (14),

$$
L_{E}=e\left\{-\frac{1}{4} F^{i j} F_{i j}+J^{k} A_{k}-\frac{2 b}{3 \kappa} \nabla_{k} A^{k}+C^{k}\left(S_{k}-b A_{k}\right)\right\}
$$

In the next section we find the variation of the total Lagrangian.

\section{VARIATION OF LAGRANGIAN FOR CHARGED, PERFECT FLUID IN RC SPACETIME}

The total Lagrangian for a charged perfect fluid with spin density is the combination of Lagrangians given by equations (2), (3), and (22):

$$
\begin{aligned}
L= & L_{G}+L_{F}+L_{E} \\
= & e\left\{\mathscr{R} / 2 \kappa-\rho\left[1+\varepsilon\left(\rho, s, F_{i j}\right)\right]-\lambda_{2} \stackrel{*}{V}_{i}\left(\rho u^{i}\right)+\lambda_{3} u^{k} \partial_{k} X\right. \\
& +\lambda_{4} u^{k} \partial_{k} s+\lambda_{1}\left(g_{i j} u^{i} u^{j}+1\right)-\frac{1}{4} F^{i j} F_{i j} \\
& \left.+J^{k} A_{k}-\frac{2 b}{3 \kappa} \nabla_{k} A^{k}+C^{k}\left(S_{k}-b A_{k}\right)\right\}
\end{aligned}
$$


where the variables of variation are $g_{i j}, \hat{S}_{i j}{ }^{k}, S_{k}, \rho, s, X, u^{i}, A_{k}$, the various Lagrange multipliers, $\lambda$, and $C^{k}$. In order to complete the variation of $L$, we must add to this system the thermodynamic relationship for a charged perfect fluid given by equation (7).

The total Lagrangian (23) yields the following variational field equations $\delta L$ :

Metric equation $\delta g_{i j}$ :

$$
\begin{gathered}
\frac{1}{2 \kappa}\left[-G^{(i j)}+\stackrel{*}{\nabla}_{k}\left(T^{k i j}+T^{k j i}\right)\right]-\frac{1}{2} \rho(1+\varepsilon) g^{i j}+\frac{1}{2} \rho \dot{\lambda_{2}} g^{i j}+\lambda_{1} u^{i} u^{j} \\
-\frac{1}{2}\left[F^{k i} F^{j}{ }_{1}+\frac{1}{4} g^{i j} F^{k l} F_{k l}\right]-\frac{4 b}{3 \kappa}\left[S^{(i} A^{j)}-\frac{1}{2} g^{i j} A_{l} S^{\prime}\right]=0
\end{gathered}
$$

where $T_{i j}{ }^{k}=S_{i j}{ }^{k}+2 \delta_{[i}^{k} S_{j]}$ is the modified torsion tensor (Benn, 1982; Heyl et al., 1976).

Proper torsion $\delta \hat{S}_{i j}^{k}$ :

$$
-\frac{1}{\kappa}\left[\hat{S}_{k}^{i j}-\hat{S}_{k}^{i j}+\hat{S}_{k}^{j i}\right]=0
$$

Torsion vector ${ }^{3} \delta S_{k}$ :

$$
-\frac{8}{3 \kappa} S^{k}+C^{k}+\frac{4 b}{3 \kappa} A^{k}=0
$$

Vector potential $\delta A_{k}$ :

$$
-F^{i j} S_{i j}^{k}+\stackrel{*}{\nabla}_{i} F^{i k}-b C^{k}+\frac{4 b}{3 \kappa} S^{k}+q \rho u^{k}=0
$$

Mass density $\delta \rho$ :

$$
-\left(1+\varepsilon+\frac{p}{\rho}\right)+\dot{\lambda}_{2}+q u^{k} A_{k}=0
$$

Four-velocity $\delta u^{k}$ :

$$
\rho \nabla_{k} \lambda_{2}+\lambda_{3} \partial_{k} X+\lambda_{4} \partial_{k} s+2 \lambda_{1} u_{k}+q \rho A_{k}=0
$$

${ }^{3}$ As an aid to those rederiving the equations, we write equation (26) including terms that cancel:

$$
-\frac{8}{3 \kappa} S^{k}+\frac{2}{3} F^{i k} A_{i}+C^{k}+\frac{4 b}{3 \kappa} A^{k}-\frac{2}{3} F^{i k} A_{i}=0
$$


Lin $\delta X$ :

$$
\stackrel{*}{\nabla}_{k}\left(\lambda_{3} u^{k}\right)=0
$$

Entropy $\delta s$ :

$$
\stackrel{*}{\nabla}_{k}\left(\lambda_{4} u^{k}\right)+\rho T=0
$$

The Lagrange multipliers give the following constraint equations:

Orthonormality of four-velocity $\delta \lambda_{1}$ :

$$
g_{i j} \mu^{i} u^{j}=-1
$$

Conservation of particle number $\delta \lambda_{2}$ :

$$
\stackrel{*}{\nabla}_{i}\left(\rho u^{i}\right)=0
$$

Lin constraint $\delta \lambda_{3}$ :

$$
\dot{X}=0
$$

Conservation of entropy $\delta \lambda_{4}$ :

$$
\dot{s}=0
$$

Torsion constraint $\delta C^{k}$ :

$$
S_{k}=b A_{k}
$$

Equations (24)-(36) represent the raw variational field equations obtained from $L$. In the next section, we arrive at the useful form of the metric, torsion, and EM field equations.

\section{FIELD EQUATIONS FOR A CHARGED SPIN FLUID}

The proper torsion field equation is obtained from equation (25) by taking the cyclic permutation of the indices $(i j k) \rightarrow(j k i)$ and then adding to the original. We find

$$
\hat{S}_{i j}^{k}=0
$$

which identically satisfies the constraint on the gauge given by equation (17) which we discussed in Section 4 on gauge invariance. It is interesting to note that in the variation of the Lagrangian there are proper torsion contributions to equations (26)-(27) from the EM part of the Lagrangian; there is, however, a compensating piece from the variation of the internal energy via the thermodynamics given by equation (7) so that in the end, the proper torsion vanishes in the EM field equation, as we will see later in this section. Note that equation (3) is also in agreement with earlier work on the status of the proper torsion for spinning fluids in RC spacetime (Smalley and Ray, 1986), 
which substantiates the relationship between the spin density of a fluid and the proper torsion.

In order to reduce the metric equation (24) to a more recognizable form, we must substitute for the Lagrange multipliers.

Multiplying the $\delta \rho$ equation (28) by $\rho$, we find

$$
\rho \dot{\lambda_{2}}=\rho\left(1+\varepsilon+\frac{p}{\rho}\right)-q \rho u^{k} A_{k}
$$

Then multiplying the $\delta u^{k}$ equation by $u^{k}$ and substituting for $\dot{\lambda}_{2}$, we get

$$
\lambda_{1}=\frac{1}{2} \rho\left(1+\varepsilon+\frac{p}{\rho}\right)
$$

Finally, upon substituting equations (38) and (39) into equation (24), we finally obtain our form of the metric field equation

$$
G^{(i j)}-\stackrel{\nabla}{\nabla}_{k}\left(T^{k i j}+T^{k j i}\right)=\kappa T^{i j}
$$

where the symmetric energy-momentum tensor has the components

$$
T^{i j}=T_{\mathrm{M}}^{i j}+T_{\mathrm{EM}}^{i j}+T_{\mathrm{GF}}^{i j}
$$

with perfect-fluid energy-momentum tensor

$$
T_{\mathrm{M}}^{i j}=\rho\left(1+\varepsilon+\frac{p}{\rho}\right) u^{i} u^{j}+p g^{i j}
$$

with electromagnetic energy-momentum tensor

$$
T_{\mathrm{EM}}^{i j}=-\left[F^{k i} F_{k}^{j}+\frac{1}{4} g^{i j} F^{k l} F_{k l}\right]
$$

plus the gauge-fixing, energy-momentum correction tensor

$$
T_{\mathrm{GF}}^{i j}=-\frac{8}{3 \kappa}\left(S^{i} S^{j}-\frac{1}{2} g^{i j} S^{k} S_{k}\right)
$$

Although this gauge-fixing term seems surprising at first, its origin derives from the requirement of overall gauge invariance of the total Lagrangian. Writing out the left-hand side of equation (40) in terms of its Riemannian plus proper torsion and torsion vector parts

$$
\begin{aligned}
G^{(i j)}-2 \vec{\nabla}_{k} T^{k(i j)}= & G^{i j}(\{\})+2 \hat{S}_{l x}^{(i} \hat{S}^{j) l x}+\hat{S}_{l x}^{(i} \hat{S}^{j) x l} \\
& -\hat{S}^{x l(i} \hat{S}_{x l}{ }^{j)}-\frac{1}{2} g^{i j}\left(\hat{S}^{l m x} \hat{S}_{l m x}+2 \hat{S}^{l m x} \hat{S}_{l x m}\right) \\
& -\frac{8}{3}\left(S^{i} S^{j}-\frac{1}{2} g^{i j} S_{k} S^{k}\right)
\end{aligned}
$$


we see that the gauge-fixing terms exactly cancel the torsion vector part, leaving only the Einstein tensor plus the proper torsion squared terms, which also vanish according to the field equation (37).

Upon multiplying the $\delta S_{k}$ equation (26) by $b$, adding to the $\delta A_{k}$ equation (27), and simplifying the covariant derivatives, we find the EM field equation

$$
\nabla_{i}^{\{\}} F^{k i}=J^{k}
$$

where $\nabla_{i}^{\{\}}$is the Riemannian covariant derivative.

The remarkable simplicity of the EM field equation (46) will be exploited in the next section in the discussion of charge conservation.

\section{PROPERTIES OF EM FIELD IN RC SPACETIME FOR PERFECT FLUIDS}

\subsection{Charge Conservation}

From the form of $J^{k}=q \rho u^{k}$, we know a priori that $\stackrel{*}{\nabla}_{k} J^{k}=0$ because the particle number conservation constraint equation (34). However, for any vector $V^{k}$ in $\mathrm{RC}$ spacetime, one can show that

$$
\stackrel{*}{\nabla}_{k} V^{k}=\nabla_{k}^{\{\}} V^{k}
$$

Then the divergence terms in equation (46) satisfy the condition (for example)

$$
\stackrel{*}{\nabla}_{k} \nabla_{i}^{\{\}} F^{k i}=\nabla_{[k}^{\{\}} \nabla_{i]}^{\{\}} F^{k i}=\frac{1}{2} K_{k i r}^{k} F^{r i}+\frac{1}{2} K_{k i r}^{i} F^{k r}=0
$$

where $K_{i j k}{ }^{l}$ is the Riemann tensor in Riemannian spacetime. Thus equation (46) under the action of the "star" derivative $\vec{\nabla}_{k}$ in RC spacetime satisfies the condition for charge conservation even though the EM field $F_{i j}$ contains explicit torsion terms.

We now turn to a discussion in the next subsection about the conditions imposed by the EM field on the EM potentials.

\subsection{Free Field Equations}

In the preceding subsection, we showed that the EM field satisfies the usual condition for charge conservation. To complete the discussion of the EM field, we must consider the constraints on the potentials obtained from the dual field equations for Div $\mathbf{B}$ and Curl $\mathbf{E}$, i.e., the "free field equations." In an inertial frame, these equations take the usual simple form Div $\mathbf{B}=0$ 
and Curl $\mathbf{E}=-\partial \mathbf{B} / \partial t$. Because of the presence of torsion, it is necessary to check these relations. We write the dual field

$$
{ }^{*} F^{i j}=\frac{1}{2} \varepsilon^{i j k l} F_{k l}
$$

where ${ }^{*} F^{i j}$ is the dual EM field tensor and $\varepsilon^{i j k l}$ is the totally antisymmetric Levi-Civita tensor. For consistency with equation (48), we take the "star" divergence of equation (49),

$$
\stackrel{*}{\nabla}_{j}^{*} F^{i j}=\nabla_{j}^{\{\} *} F^{i j}+{ }^{*} F^{k j} \hat{S}_{j k}{ }^{i}=\stackrel{*}{\nabla}\{\} * F^{i j}
$$

where the last step follows because of the proper torsion field, equation (37). Upon substituting equations (6) and (49) into the above equations and using the field equations (36) and (37), we obtain

$$
\stackrel{*}{*}_{k}^{*} F^{i j}=\varepsilon^{i j k l} \nabla_{[j}^{\{\}} \nabla_{k}^{\{\}} A_{l]}=\varepsilon^{i j k l}\left[-\frac{1}{2} K_{[j k l]}{ }^{x} A_{x}\right]=0
$$

where in the last step we used the circulating identity for the Riemann curvature tensor in Riemannian spacetime (Schouten, 1954). Thus, one obtains the usual free field equations.

It is interesting to note that in this application, we find that the torsion vector is a propagating field proportional to the EM vector potential. If this theory is further constrained to the case where there is no EM field, then particle number conservation is sufficient to remove the torsion vector. Then this is just the case of a perfect fluid in general relativity (Ray and Smalley, 1982a, 1983a,b; Ray, 1972).

\section{CONCLUSIONS}

We have shown that it is possible to find a variational theory in $\mathrm{RC}$ spacetime for a charged perfect fluid in which the EM field is minimally coupled to the geometry through the RC connection [see equation (6)]. We found that it was necessary to extend the self-consistent formulation for perfect fluids by requiring that the total Lagrangian for the theory be gauge invariant under the extended gauge transformation given by equations (8) and (9). This requires that there be a constitutive relationship between the torsion vector and the EM vector potential plus the addition of a gaugefixing term to the total Lagrangian.

With the above restrictions, we find that the field equation for the EM field takes the very simple form given by equation (46), which is the same form that one finds in the Einstein-Maxwell theory in Riemannian spacetime, or that one finds in the Einstein-Cartan-Maxwell theories in which one assumes the two-form approach for the EM field as opposed to the minimal coupling approach that we assume in this work. Here the field tensor $F^{i j}$ in equation (46) contains specific torsion terms. However, due to 
the various field equations in the theory, we find that the EM field, as described by equation (46), conserves charge, obeys particle number conservation, and gives the usual free field equations. In this development, the EM field specifically couples to the RC geometry and directly influences the perfect-fluid energy-momentum. In effect, the overall theory does not neglect the obvious interaction of the EM field on the fluid density distribution and the consequent change in energy-momentum. The origin of this effect is in the extended thermodynamics given by equation (7), the conservation of particle number, and the constraint of the torsion vector to be proportional to the vector potential of the EM field.

The metric field equation gives a symmetric energy-momentum tensor that now includes an internal energy directly dependent on the EM field; a symmetric EM energy-momentum; and a (symmetric) gauge-fixing term.

We have demonstrated a new way that one can couple the EM field to $\mathrm{RC}$ spacetime, while retaining the expected properties of the EM field in the presence of a gravitational field. Our particular method treats the EM field as part of the overall system instead of as an external field as in developments where the EM field is a two-form. We have a well-defined theory of the interaction of the EM field with a perfect fluid in RC spacetime which has as its limit the Lagrangian-based theory with particle number conservation of a perfect fluid in RC spacetime when the EM field vanishes.

Elsewhere we consider the highly interesting extension of the present work to perfect fluids with spin density. In this case, the proper torsion field would be active because of the spin density of the fluid.

\section{REFERENCES}

Amorim, R. (1984). Physics Letters, 104A, 259.

Amorim, R. (1985). Physical Review D, 31, 3099.

Benn, I. M. (1982). Annales de l'Institut Henri Poincaré, 37, 67.

De Ritis, R., Lavorgna, M., Platania, G., and Stornaiola, C. (1985). Physical Review D, 31, 1854.

Dirac, P. A. M. (1959). Physical Review D, 114, 929.

Fock, V. (1964). The Theory of Space Time and Gravitation, Pergamon, Macmillan, New York. Grensing, D., and Grensing, G. (1983a). Physical Review D, 28, 286.

Grensing, D., and Grensing, G. (1983b). General Relativity and Gravitation, 15, 985.

Guggenheim, E. A. (1936). Proc. Roy. Soc. A, 20, 49, 70.

Hammond, R. T. (1988). General Relativity and Gravitation, $20,813$.

Hammond, R. T. (1989). Classical and Quantum Gravity, 6, L195.

Hehl, F. W. (1973). General Relativity and Gravitation, 4, 333.

HehI, F. W. (1974). General Relativity and Gravitation, 5, 491.

Heyl, F. W., von der Hyde, P., and Kerlick, G. D. (1976). Review of Modern Physics, 48, 393.

Hojman, S., Rosenbaum, M., Ryan, M. P., and Shepley, L. C. (1978). Physical Review D, 17, 3141. 
Messiah, A. (1962). Quantum Mechanics, Vol. II, North-Holland, Amsterdam, p. 918.

Mukku, C., and Sayed, W. A. (1979). Physics Letters, 82B, 382.

Novello, M. (1976). Physics Letters, 59A, 105.

Ray, J. R. (1972). Journal of Mathematical Physics, 13, 1451.

Ray, J. R., and Smalley, L. L. (1982a). Physical Review Letters, 49, 1059.

Ray, J. R., and Smalley, L. L. (1982b). Physical Review D, 26, 2615.

Ray, J. R., and Smalley, L. L. (1983a). Physical Review D, 27, 1383.

Ray, J. R., and Smalley, L. L. (1983b). Physical Review Letters, 50, 626E.

Schouten, J. A. (1954). Ricci Calculus, 2nd ed., Springer-Verlag, Berlin, p. 144.

Smalley, L. L. (1986). Physical Review D, 33, 3590.

Smalley, L. L., and Ray, J. R. (1986). General Relativity and Gravitation, 18, 549.

Spinosa, R. (1987). Physics Letters, 125A, 228. 